\title{
Measuring the Electrical Stapedius Reflex with Stapedius Muscle Electromyogram Recordings
}

\author{
Ryan S. Clement, ${ }^{1}$ PAUl M. CARTER, ${ }^{2}$ and DARYl R. KIPKE ${ }^{3}$ \\ ${ }^{1}$ Department of Bioengineering, Arizona State University, Tempe, AZ; ${ }^{2}$ Cochlear Corporation, Englewood, CO; \\ and ${ }^{3}$ Department of Biomedical Engineering, University of Michigan, Ann Arbor, MI
}

(Received 27 February 2001; accepted for publication 19 September 2001)

\begin{abstract}
Previous studies have demonstrated a correlation between cochlear implant recipients' comfort levels $(C$ level, upper limit of dynamic range of stimulation) and the contralateral electrical stapedius reflex (ESR) threshold, detected by acoustic impedance change. However, the utility of the approach is limited because many recipients have no detectable impedance change. The goals of this study were to investigate the utility of the stapedial electromyogram (EMG) for estimating onset and strength of the ESR. Ketamine-anesthetized guinea pigs were implanted with Nucleus electrode arrays and stimulated with biphasic current pulse trains (250 pps) via a Cochlear Corporation CI24M stimulator. Typical EMG recordings (obtained with bipolar microwire electrodes) contained easily detectable unit potentials up to $300 \mu \mathrm{V}$ in amplitude. Growth response curves (obtained from threshold-crossing counts or rms of the EMG signal) were typically monotonic with dynamic ranges spanning $700 \mu \mathrm{A}$ or $8 \mathrm{~dB}$. Based on adaptation and temporal properties, the stimulus protocol (500 ms duration with 4-5 s interstimulus intervals) was adequate for producing independent responses. The data presented are consistent with ESR characteristics (acoustic impedance technique) of cochlear implant recipients and with EMG properties of acoustically stimulated guinea pigs. Use of the EMG for characterizing the ESR may eventually be applied to human cochlear implant recipients as a guide in setting the upper limit of the dynamic range. (C) 2002 Biomedical Engineering Society. [DOI: 10.1114/1.1454132]
\end{abstract}

Keywords-Cochlear implants, Comfort level ( $C$ level), Electrophysiology, Microwire electrodes, Guinea pigs.

\section{INTRODUCTION}

Accurately establishing dynamic ranges of electrical stimulation is critically important in fitting cochlear implants. Currently, these are estimated clinically using time-consuming protocols that require subjective responses from the patient. ${ }^{22}$ These procedures are especially troublesome for very young children who may not have a well-defined concept of loudness and lack sufficient communication abilities. ${ }^{26}$ Objective electrophysi-

Address correspondence to: Daryl R. Kipke, Department of Biomedical Engineering, 3304 G.G. Brown, 2350 Hayward, University of Michigan, Ann Arbor, MI 48109-2125. Electronic mail: dkipke@umich.edu ological measurements such as the electrical compound action potential (EAP), the electrical auditory brainstem response (EABR), and the electrical stapedius reflex (ESR) can aid the clinician in difficult cases. ${ }^{23,24}$ In the future, these types of measurements might become commonplace in the cochlear implant fitting procedure. ${ }^{32}$ The present study is concerned with the ESR, which has the potential to serve as an objective aid for setting the high end of the dynamic range (the "comfort level," or $C$ level). . $^{3,14,17,25,28}$

The stapedius muscle attaches to the stapes, the last of three tiny bones in the middle ear that transfer sound energy from the eardrum to the cochlea. It is the smallest striated muscle in the body, averaging, in adults, 6-7 $\mathrm{mm}(4-5 \mathrm{~mm}$ in neonates) in length by $2-3 \mathrm{~mm}$ in diameter, ${ }^{4}$ and only $0.75 \mathrm{~mm}$ by $0.3 \mathrm{~mm}$ in the guinea pig. ${ }^{7}$ The muscle is located very close to the facial nerve. It originates in the periosteum of the stapedial cavity that encases most of the muscle. Through a small slit in the pyramidal eminence structure, the tendon of the stapedius exits to the middle ear and inserts onto the neck of the stapes (in the guinea pig a significant portion of the muscle is exposed in addition to the tendon). The muscle fibers converge from the posterior portion of the stapedial cavity to attach to the central tendon and are 8-14 $\mu \mathrm{m}$ in diameter in adults, and $6 \mu \mathrm{m}$ or less in neonates ${ }^{5}$ and guinea pigs. ${ }^{7}$ The number of muscle fibers estimated for each motor unit is extremely low (less than 9 in adults, ${ }^{4}$ around $16-21$ in neonates, ${ }^{5}$ and $14-19$ in guinea $\left.\mathrm{pigs}^{7}\right)$. Due to its extremely small size and low innervation ratio, the stapedius is unique among other skeletal muscles. At suprathreshold stimulation (acoustic or electric), the stapedius muscle contracts in nearly isometric fashion.

The contractions of the stapedius muscle increase the mechanical rigidity of the bony chain and alter the transfer function of the middle ear. ${ }^{6}$ Thus, the activation of the stapedial reflex can usually be detected indirectly and noninvasively using an electroacoustic impedance bridge to measure the increase in acoustic impedance seen at 
the tympanic membrane. ${ }^{37}$ Contralateral acoustic impedance measurements were used in several studies to characterize growth response relationships and demonstrate a strong correlation between the threshold of the ESR and the behaviorally established comfort levels for cochlear electrodes. 3,15,17,25,27,28 Acoustic impedance-based approaches for estimating comfort levels may have limited clinical utility because several studies reported that approximately $30 \%$ of surveyed patients did not exhibit impedance changes. ${ }^{3,15,25,27}$ Other authors have reported the ability to record the response in nearly all subjects, but most of these studies prescreened for middle ear abnormalities which might be present in many cochlear implant recipients. ${ }^{17,28,29}$ The success of the acoustic impedance-based approach also relies heavily on patient cooperation in keeping the impedance probe sealed in the ear canal for lengthy amounts of time, which may not be well tolerated by children. ${ }^{25}$

The stapedial muscle electromyogram (EMG) provides an alternative albeit invasive, method of detecting the stapedial reflex. The acoustically elicited reflex has been studied using stapedial EMG in several animal models. ${ }^{2,9,21}$ In a study using ketamine-anesthetized guinea pigs, Avan et al. found that the sharp onset and lack of spontaneous activity in the stapedial EMG made it a good estimator of reflex threshold. ${ }^{2}$ In human subjects with unilateral ear drum perforation, Zakrisson et al. found that the contralateral integrated EMG growth response and the ipsilateral acoustic impedance response curves were similar (differing by less than $6 \mathrm{~dB}$ on average), with the EMG having a slightly more sensitive threshold. ${ }^{37}$ Almqvist et al. obtained ipsilateral averagedEMG stapedial reflex (AESR) recordings intraoperatively in children and demonstrated a close correspondence to postoperative contralateral acoustic impedance thresholds and behavioral comfort levels for the apical channels in one patient. ${ }^{1}$ In that study, averaged-EMG recordings were obtained after brief stimulus bursts (1-4 pulses) using a relatively large monopolar recording electrode.

The objectives of the present study were to investigate the utility of using ipsilateral stapedial EMG recordings for estimating the threshold, strength, and time course of the ESR for a given set of stimulation parameters. The overall approach was to implant cochlear electrodes in anesthetized guinea pigs and record the stapedial EMG in response to pulse-train stimuli that were comparable to those used in fitting cochlear implants. A relatively simple bipolar microwire EMG electrode was designed and placed directly onto the stapedius. Two signal processing algorithms were evaluated to assess the strength of the reflex and to obtain growth functions that could be compared with the visual threshold of muscle contraction. The growth functions and temporal characteristics of the ESR obtained from the analyzed EMG were qualitatively compared to acoustic impedance results from human cochlear implant recipients reported in the literature, and with stapedial EMG recordings obtained in acoustically stimulated guinea pigs by Avan et al. ${ }^{2}$

\section{METHODS}

\section{Surgical Procedures}

Four female Sprague-Hawley guinea pigs (GP22GP26) weighing 600-900 g, were initially anesthetized with a mixture of $30 \mathrm{mg} / \mathrm{kg}$ of ketamine and $6 \mathrm{mg} / \mathrm{kg}$ xylazine and supplemented regularly to maintain areflexia during the experimental procedure. The physiological state of the animal was monitored using blood oxygen saturation and heart rate. The animal was placed on a heating blanket maintained at $37^{\circ} \mathrm{C}$ and given an oxygen-rich air mixture to breathe. On occasion, atropine (60 $\mu \mathrm{g} / \mathrm{kg}$ ) was delivered intramuscularly to counter the cardiodepressive action of the anesthetic. All animal procedures followed NIH guidelines and were approved by the Arizona State University Institutional Animal Care and Use Committee.

The skin and fascia covering the cranium were removed. The head was fixated to a flex-bar clamp using a bolt placed on the exposed cranium and held with several bone screws and dental acrylic. The pinna and the external ear canal of the operated ear were completely removed to expose the outer surface of the tympanic bulla. The bony portion of the external ear canal was ground flat and widened with a surgical drill until the eardrum was almost completely exposed. The eardrum was pierced and partially removed with the tip of a hypodermic needle. The stapedius muscle, incu-stapedial joint, and round window of the cochlea was visualized using a surgical microscope.

The apical segment of a Nucleus cochlear implant electrode array (Cochlear Corporation, Englewood, CO) was carefully inserted through an accessory hole drilled in the superior tympanic bulla and guided with an insertion tool into the cochlea via the round window (after slight perforation of the membrane with a hypodermic needle). The Nucleus cochlear implant electrode array is composed of banded $\mathrm{Pt}-\mathrm{Ir}$ electrodes wrapped around a silastic carrier. The diameter of the inserted portion of the implant was $450 \mu \mathrm{m}$, and each electrode band was $300 \mu \mathrm{m}$ thick with each separated from one another by $750 \mu \mathrm{m}$. The insertion depth (5-8 electrode bands) was determined from review of the videotaped insertion procedure. For all the data presented in this study, the stimulation was always provided on the most apical channel with one inactive electrode between the stimulating electrodes $(1.5 \mathrm{~mm}$ electrode separation; Cochlear Corporation's BP +1 stimulation mode). Implant placement and function was verified using standard electrical impedance 
measurements. Electrical stimulation was verified to reliably activate the stapedial reflex in all subjects in this study.

\section{Stimulation and Recording}

The cochlea was stimulated using biphasic pulse trains through a Nucleus CI24M cochlear implant system (Cochlear Corporation). Pulses had a $100 \mu$ s per phase width and a repetition rate of $250 \mathrm{~Hz}$. The current was varied up to $1750 \mu \mathrm{A}$. Unless otherwise stated, bursts of $500 \mathrm{~ms}$ duration were applied. Typically, the time between stimulus bursts in a series [interstimulus interval (ISI)] was greater than $4 \mathrm{~s}$. The clinical-grade stimulator was packaged in a small plastic box with a connector that mated to the percutaneous connector of the cochlear electrode array (Cochlear Corporation's standard “implant in a box" stimulator). The implant system was controlled in software (WINDPS VERSION R116, Cochlear Corporation). A separate trigger signal was generated by the stimulation system for synchronizing the stapedius muscle EMG records with cochlear stimulation.

The stapedial EMG electrodes were fabricated in the laboratory from polyimide-insulated 50 - $\mu \mathrm{m}$-diameter tungsten microwires. Two wires were soldered into a miniconnector and twisted together to within a few millimeters of their tips such that the tip spacing was approximately $200 \mu \mathrm{m}$. Tips were cut flush with precision scissors to yield blunt tips that would provide a surfacetype EMG recording. This approach obviated puncture of the relatively thin muscle. The typical electrical impedance range in $0.9 \% \mathrm{w} / \mathrm{v}$ saline was $30-60 \mathrm{k} \Omega$ at $1 \mathrm{kHz}$, which was similar to measured in vivo values (20-80 $\mathrm{k} \Omega$ ).

Subsequent to placement of the cochlear electrode, the EMG electrode assembly was mounted on a micromanipulator and carefully placed on the belly of the stapedius muscle through the bulla opening. The electrode tips were oriented along a line approximately parallel with the muscle axis with one electrode placed near the tendon and the other near the exit from the bone (approximately a belly-tendon-type configuration). The electrode tips were held in tight contact with the muscle surface during strong contractions by inserting the electrode until it bent slightly due to the mechanical resistance of the muscle. A 500- $\mu \mathrm{m}$-diameter uninsulated stainless-steel wire electrode was placed under the skin to provide an electrical ground point for the instrumentation.

The EMG was recorded differentially from the EMG electrodes using a commercial high-impedance preamplifier (Stanford Research Systems, CA; model SR560). The EMG was filtered (bandpass filter with cutoff frequencies of 300 and $10,000 \mathrm{~Hz}$ with $6 \mathrm{~dB} /$ octave rolloff), amplified (80 dB), and then sampled at $20 \mathrm{kHz}$ (unless otherwise noted) with a 12-bit data acquisition

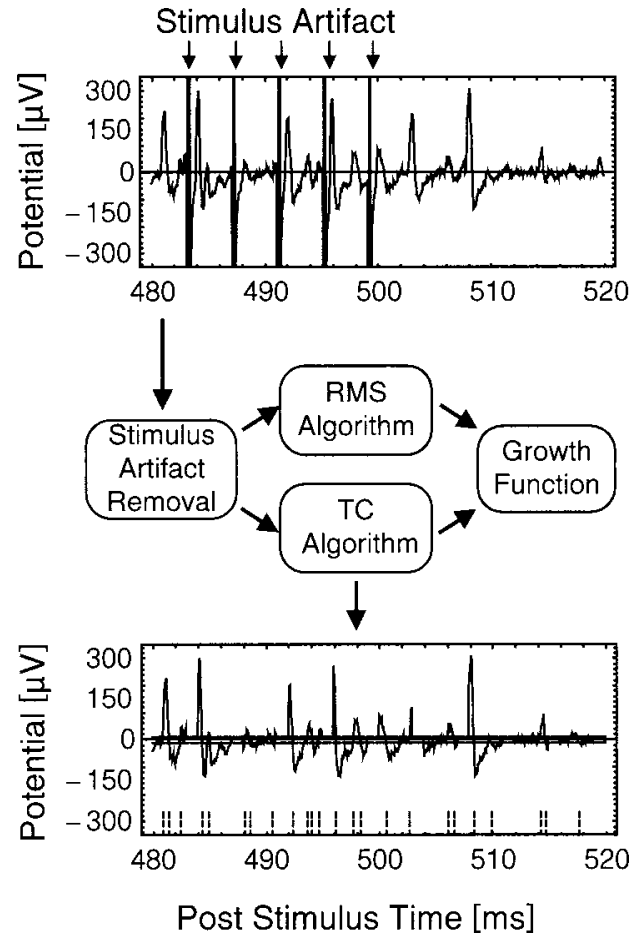

FIGURE 1. Signal processing sequence in quantifying the strength of stapedial EMG. Stimulus artifact (indicated by the arrows) was removed off-line by zeroing windows. Threshold crossings or rms assessed the strength of the EMG. Growth functions were obtained by averaging the strength at increasing stimulus levels. Vertical dashed lines on the bottom plot indicate valid threshold crossings. The thresholds for detection are the horizontal dashed lines (3 SD of the background during nonstimulus, $\pm 12 \mu \mathrm{V}$ here). (Data obtained from GP22 stimulated at $1429 \mu \mathrm{A}$.)

board for computer storage and offline analysis. Monopolar EMG recordings were also attempted but found to be problematic because of the large stimulus artifact.

\section{Data Analysis}

In order to eliminate the stimulation artifact, prior to analysis, $1 \mathrm{~ms}$ zeroing windows were applied at the time of each stimulus pulse throughout each record (Fig. 1). In addition, any dc offset was removed by forcing the mean of the non-EMG portion of the wave form to $0 \mathrm{~V}$.

In order to estimate response growth characteristics of the ESR, two separate algorithms were used to quantify the stapedial EMG. The "rms-energy" algorithm involved simply calculating the root-mean-square energy of each artifact-eliminated EMG record and subtracting the base-line rms calculated during a period when the stimulus was off. An alternative algorithm based on threshold crossings provided an additional measure of the relative strength of each response (estimated by the total number of valid threshold crossings in the entire record). This method was also used to analyze the temporal characteristics of the EMG signal. As the muscle 
becomes more active, the frequency of motor unit activity increases, which results in more threshold crossings. This algorithm is especially helpful when the EMG records consist of widely varying spikes that occur at relatively low rates, ${ }^{18}$ which is a characteristic of most EMG records in this study. The threshold-crossing algorithm involved first defining standard threshold levels at three standard deviations above and below the average base-line EMG level that was recorded during the final $200 \mathrm{~ms}$ of the records in a growth series (typically, 300-500 ms poststimulus). Second, the times of the valid threshold crossings were obtained. This process involved examining the record in $4 \mathrm{~ms}$ segments (corresponding to the rate of the stimulus pulse delivery). Following the initial $1 \mathrm{~ms}$ zeroed segment, and after the signal entered the region between the positive and negative threshold levels (in case of residual stimulation artifact), valid threshold crossings were defined as instances in which the signal crossed the threshold (positive or negative) for a detection window of at least $0.2 \mathrm{~ms}$ or four sample points (window increased to 0.4 ms for Fig. 7 because of $5 \mathrm{kHz}$ sampling rate). A maximum of 15 crossings can occur in each $3 \mathrm{~ms}$ segment $(5000 / \mathrm{s})$. However, because most motor-unit potentials are on the order of $1 \mathrm{~ms}$ and are often superimposed, the practical threshold-crossing limit is less than this. The threshold-crossing algorithm applied to a typical EMG record is illustrated in Fig. 1. A signal-to-noise ratio was estimated from the ratio of the largest amplitude EMG spikes to the computed threshold level, expressed in decibels to the nearest $5 \mathrm{~dB}$.

Response Growth. Response growth functions were constructed to summarize the threshold, dynamic range, and saturation of the ESR as a function of stimulus level. Each growth function was obtained by computing the average strength of the EMG responses (using rms or threshold-crossing algorithms) at each current level. Each response record was $1 \mathrm{~s}$ in duration with $500 \mathrm{~ms}$ during the stimulus and $500 \mathrm{~ms}$ after the stimulus. For three of the animals (GP24-GP26) the current levels were incremented for each stimulus presentation and the whole series was presented a number of times (2-6). The growth function from GP22 was obtained by presenting the same current level ten times before moving to the next current level. Normalized growth functions were obtained by dividing each data point by the strongest response in the computed growth function. Threshold and saturated were defined as $10 \%$ and $90 \%$ of maximum response, respectively. The dynamic range (expressed in $\mu \mathrm{A}$ or $\mathrm{dB}$ ) was calculated from these two levels.

Temporal Response Properties. The latency to the first threshold crossing (TC) was used to estimate the ESR latency as a function of current level. To examine additional temporal features, the threshold-crossing times were binned at either $12 \mathrm{~ms}$ for $500 \mathrm{~ms}$ stimuli or $40 \mathrm{~ms}$ for 2-3 s stimuli and used to construct histograms to summarize the stimulus-locked average EMG response. Each bin was normalized to threshold crossings per second by dividing the total number of counts in the bin by the total time for that bin. The total time for each bin was calculated by multiplying the bin width by the number of presentations and correcting by a factor of 0.75 , because only $3 \mathrm{~ms}$ out of $4 \mathrm{~ms}$ were available for counting after the start of each stimulus pulse due to the $1 \mathrm{~ms}$ zeroing windows. In one animal (GP25), adaptation to long duration $(2-4 \mathrm{~s})$ was investigated by histograms of the threshold crossings of a series of ten presentations to verify known adaptation features of the stapedius muscle to significantly long stimuli. In the same animal, the effect of interstimulus interval upon adaptation of EMG strength to repetitive stimuli $(N=50)$ was investigated by presenting a series of $500 \mathrm{~ms}$ stimulus bursts with various ISIs $(0.5,1,2$, and $4 \mathrm{~s})$ at two different current levels to determine the minimum ISI required for obtaining independent ESR responses.

\section{RESULTS}

Data are presented from intraoperative EMG recordings from four guinea pigs. Each EMG record was initiated at the onset of stimulation and continued for several hundred milliseconds after the simulation ceased (typically, $500 \mathrm{~ms}$ stimulus on, $500 \mathrm{~ms}$ stimulus off).

\section{EMG Recordings}

Typically EMG recordings contained single- and multiple-motor-unit potentials of varying amplitudes up to $300 \mu \mathrm{V}$ (all animals had potentials $>50 \mu \mathrm{V}$ in their records) with estimated signal-to-noise ratios between 10 and $25 \mathrm{~dB}$ (Fig. 2). The EMG spike frequency increased with the stimulus current level, but the maximum amplitudes of the spikes remained relatively constant. For sufficiently high stimulus levels, EMG activity persisted for 100-400 ms after the stimulus ended. There were no spontaneous EMG spikes after this response dissipated or whenever a suprathreshold stimulus was absent. Subthreshold stimuli were defined as those current levels that did not elicit EMG spikes (424 $\mu \mathrm{A}$, in example). Consistent with the wave forms in Fig. 1, the potentials in each animal had similar polarity, which suggests that they were detected by only one of the electrodes. This characteristic is expected with a belly-tendon electrode placement. 


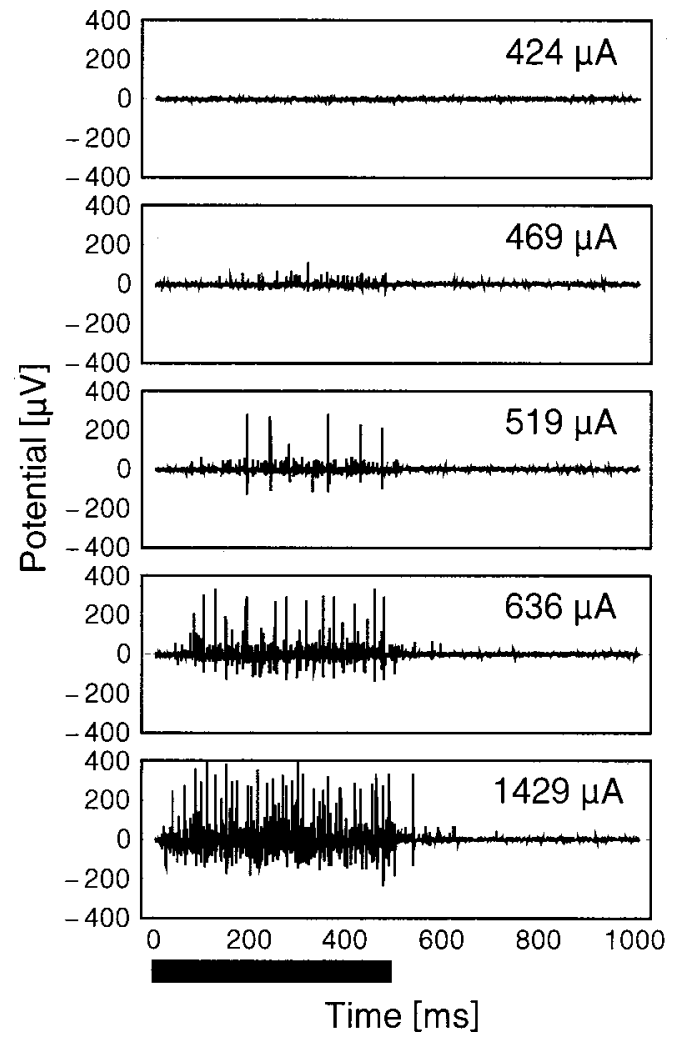

FIGURE 2. Representative EMG responses (after artifact removal) to increasing current level for one subject (GP22). The horizontal bar at the bottom indicates stimulus on (500 ms). Current level is specified in the upper-right corner of each plot. The same scaling was used for all plots with the $x$ axis in "ms" and the $y$ axis in " $\mu \mathrm{V}$."

\section{Response Growth Functions}

The response growth of the stapedial EMG activity as a function of current level was typically monotonic with a dynamic range of approximately $700 \mu \mathrm{A}$ or $8 \mathrm{~dB}$ (rms: $753 \mu \mathrm{A}$ or $8.2 \mathrm{~dB}$; TC: $675 \mu \mathrm{A}$ or $7.7 \mathrm{~dB}$ ) (see Fig. 3). The visual thresholds (arrows) of muscle contraction were near the lower end of the transitions from threshold to saturation (around $500 \mu \mathrm{A}$ for most animals). The threshold-crossing and rms-energy algorithms resulted in similar normalized growth response curves for each animal $(r>0.97)$ with thresholds varying by less than $1 \mathrm{~dB}$ and saturation varying by less than $2 \mathrm{~dB}$. For one animal (GP24), decreased EMG spike amplitudes resulted in a decrease in the rms-energy growth function at stimulus levels higher than $1200 \mu \mathrm{A}$. This loss in signal-to-noise ratio might be attributed to the electrode losing tight contact with the muscle during strong contractions. The threshold-crossing algorithm was more robust to this type of signal degradation. Reliable response growth functions could be obtained by averaging over relatively few stimulus presentations at each current level $(N$ $=2-10)$.
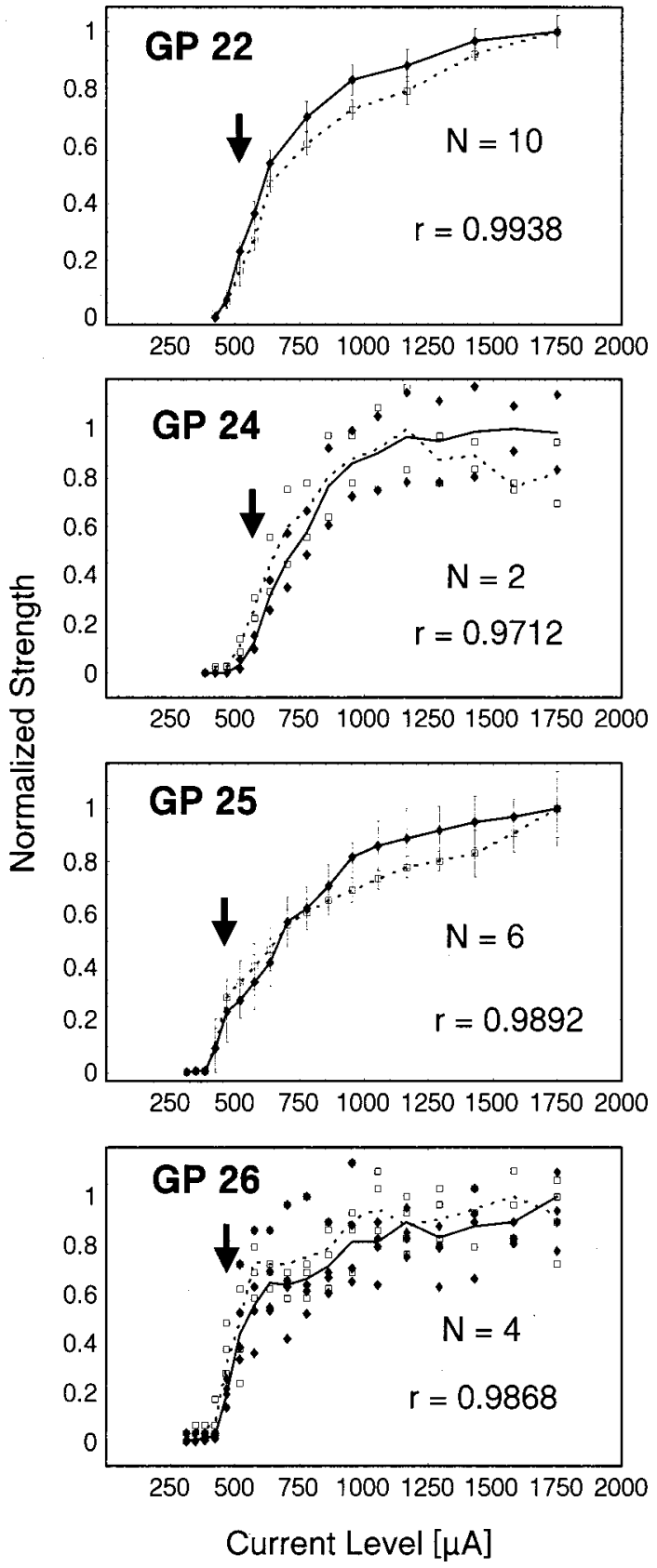

FIGURE 3. Normalized response growth functions for all four subjects. The responses are normalized by the greatest response in each series. Arrows indicate visual detection of contraction. Diamonds/solid line: obtained by the thresholdcrossing algorithm. Open boxes/dashed line: obtained by the rms-energy algorithm. Correlation coefficients $(r)$ between rms and threshold-crossing algorithm growth functions are indicated for each plot. $\boldsymbol{N}$ refers to the number of averages used to obtain each data point in the individual plots, with the error bars indicating the associated standard deviation (actual values shown for GP24 and GP26 because $N \leqslant 4$ ).

While the level of the saturated EMG differed across animals [Fig. 4(A)], the normalized growth functions had a consistent shape and dynamic range [Fig. 4(B)]. The 

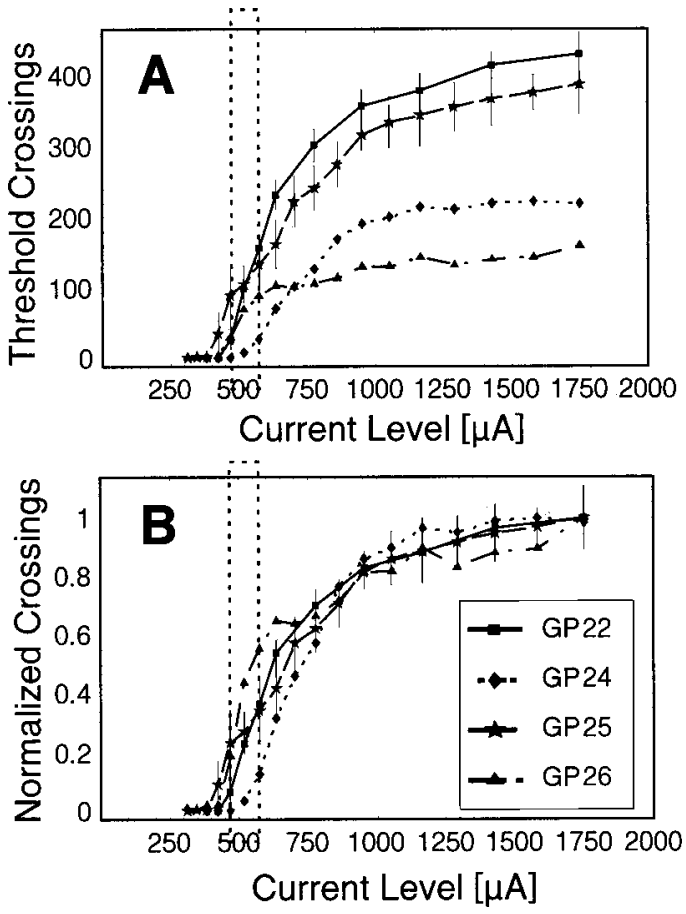

FIGURE 4. Comparison of response growth functions from different subjects, obtained by the threshold-crossing algorithm. (A) Un-normalized; (b) normalized. The vertical box encompasses the range of the visually detected ESR thresholds. (Same data set as Fig. 3.)

one exception (GP24) had essentially the same dynamic range but the response curve was shifted right about 200 $\mu \mathrm{A}$. In this animal only six electrode bands were inserted into the cochlea as compared to eight bands in GP25. It was observed that moving the stimulation to the basal channels of the cochlear electrode shifted the growth functions in a similar manner (also observed by Battmer et $a .^{3}$ in humans). Therefore, the deviation observed in GP24 may be accounted for by more basal stimulation due to a shorter cochlear electrode insertion.

\section{Temporal Response Properties}

Significant temporal integration of the EMG was revealed by stimulus synchronized poststimulus time histograms (PSTHs) (Fig. 5). The latency to the maximum EMG response decreased from approximately $200 \mathrm{~ms}$ near the threshold stimulus $(469 \mu \mathrm{A})$ to less than $50 \mathrm{~ms}$ at high stimulus levels that elicited a saturated EMG response $(1429 \mu \mathrm{A})$. The EMG activity remained at a maximum until the stimulus was turned off. In all animals, at sufficiently high current levels, the response diminished to zero over $100-400 \mathrm{~ms}$ following the stimulus cessation.

The average latency to the first threshold crossing was relatively large (100-400 ms) with high variability near the ESR threshold (Fig. 6). In contrast, the latency at

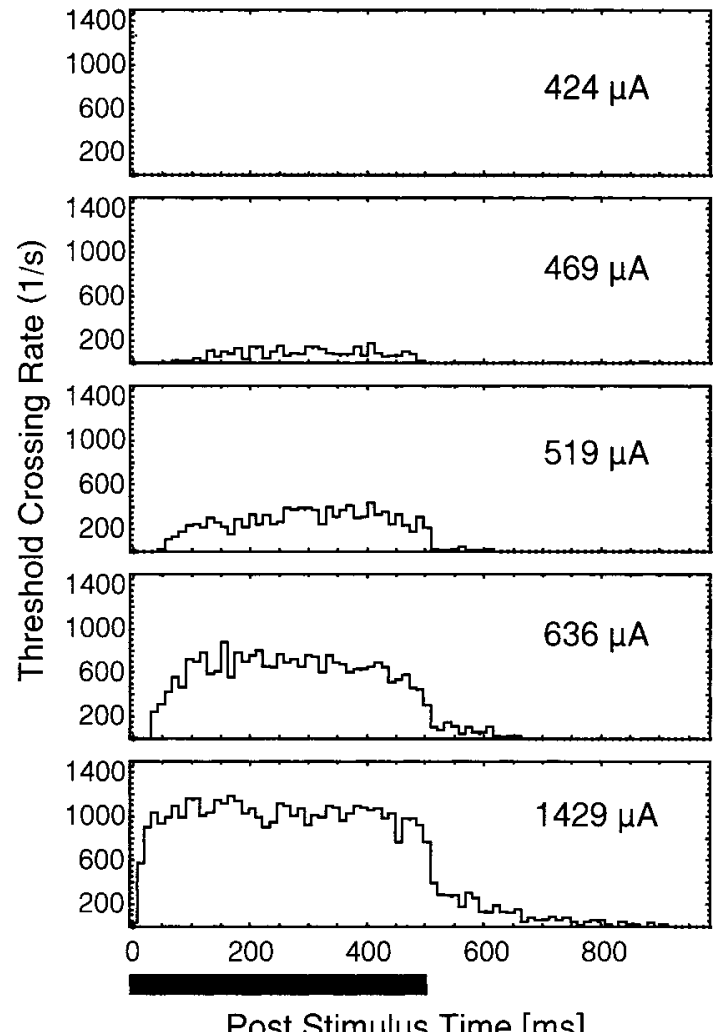

Post Stimulus Time [ms]

FIGURE 5. Representative poststimulus time histograms (PSTHs) of crossings detected by the threshold-crossing algorithm reveal temporal integration (GP22). The vertical axes are the threshold-crossing rates for each bin (bin width: 12 ms) after ten presentations, time corrected for zeroing windows (see the Methods section). The horizontal axis is the time passing after stimulus onset is in " $m s$." The thick black bar indicates the stimulus on. The stimuli current level increases from top to bottom and is indicated at the upperright corner of each plot (same current level as used in Fig. 2).

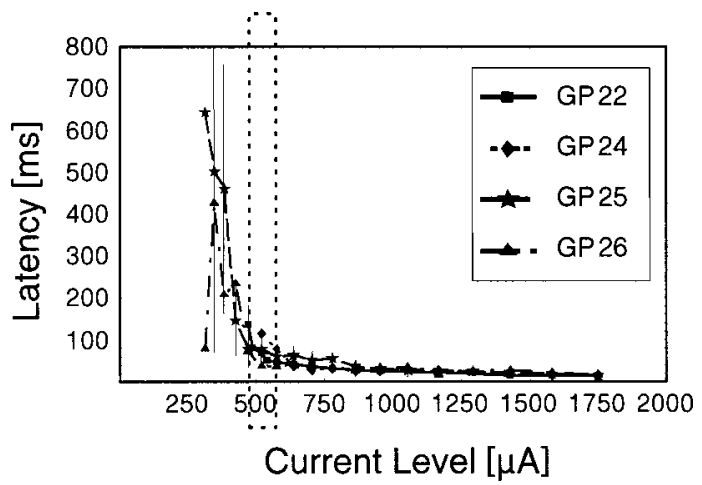

FIGURE 6. Average latency of the first threshold crossing for all four subjects as a function of stimulus current level. Vertical box indicates visual detection range of contraction. The bars associated with each data point show the standard deviation (same data set as in Fig. 3). 


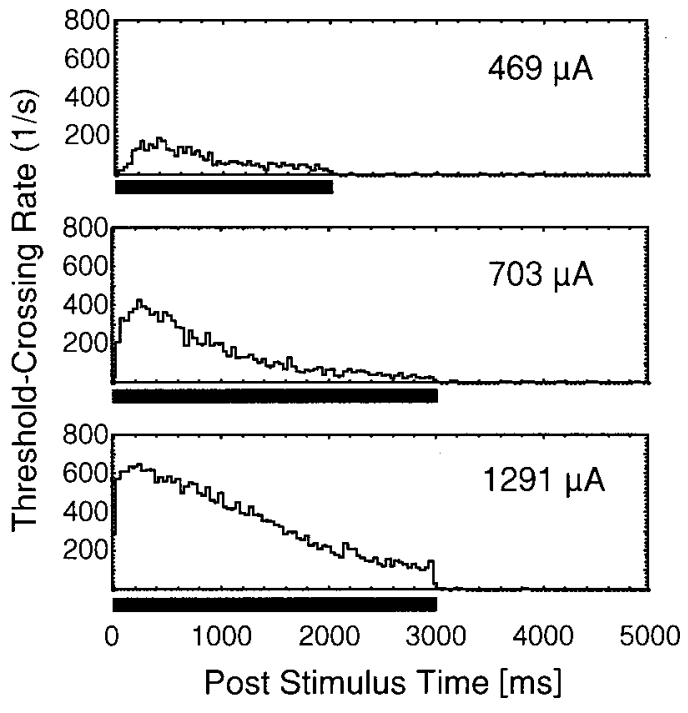

FIGURE 7. Poststimulus time histograms for long-duration stimuli for one subject (GP25). The vertical axis is the threshold-crossing rate for each bin (bin width: $\mathbf{4 0} \mathrm{ms}$ ) after ten stimulus presentations, time corrected for zeroing window (see the Methods section). The horizontal axis is the time lapsed from stimulus onset in "ms." The bar below each plot indicates the stimulus on and the current level is shown in the upper-right corners. (The sampling rate of data acquisition was changed to $5 \mathrm{kHz}$.)

simulation levels that elicited a saturated response was between 15 and $20 \mathrm{~ms}$ with much lower variability among animals. The transition region was roughly the same as that for the EMG growth functions, which demonstrates that less temporal summation is required for higher stimulus levels.

\section{Response Adaptation}

Adaptation of the EMG response occurred after $1 \mathrm{~s}$ of stimulation (Fig. 7). This was true for stimulus levels near threshold $(469 \mu \mathrm{A})$, near the middle of the dynamic range $(703 \mu \mathrm{A})$, and at levels eliciting a saturated response $(1291 \mu \mathrm{A})$. At current levels of 469 and $703 \mu \mathrm{A}$, the adaptation was nearly complete before the stimulus ended, while at $1291 \mu \mathrm{A}$ the response decayed to a base-line level. The sustained base-line level at $1291 \mu \mathrm{A}$ was found to be present only in the first few records in the series used to compute the average response (ten total records). In contrast, the latter records exhibited more complete adaptation, similar to the responses at the lower current levels. Therefore, it is apparent that stimulus amplitude effects the degree of adaptation to subsequent stimuli, with higher amplitudes resisting adaptation.

Decreasing the interstimulus interval also caused significant adaptation to repetitive stimulation at the same level (Fig. 8). This was true for stimulus levels at the lower end of the dynamic range $(574 \mu \mathrm{A})$ as well as for

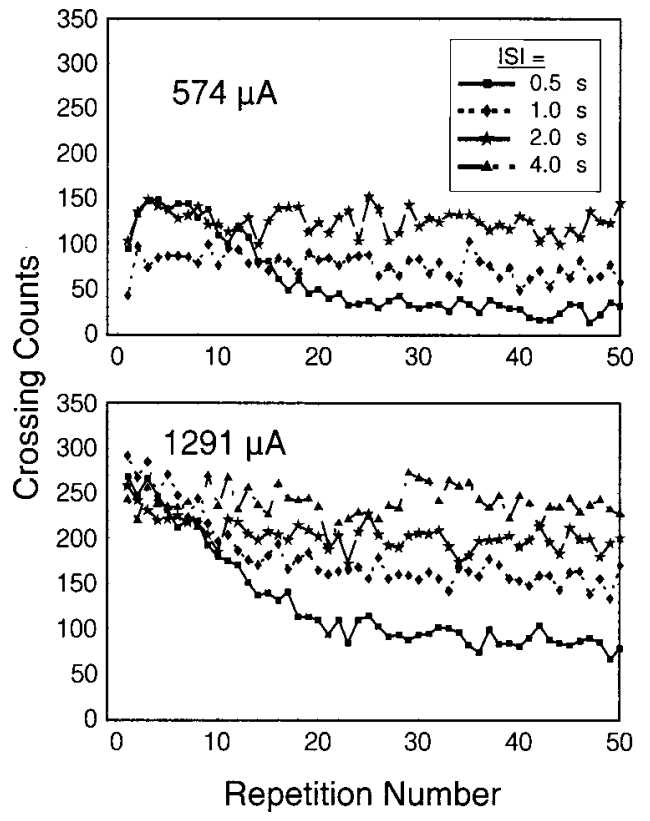

FIGURE 8. Effect of interstimulus interval (ISI) on the stapedius muscle EMG activity in one subject (GP25). The vertical axis is the total number of crossing counts obtained by the threshold-crossing algorithm for each stimulus presentation (500 ms duration). The horizontal axis is the repetition number. The current levels are indicated in the upper-left corners. The ISI used for each plot is indicated by the legend. (The sampling rate for EMG records was $10 \mathrm{kHz}$.)

saturation levels $(1291 \mu \mathrm{A})$. Similar to the results from long duration stimuli, more adaptation is observed at the lower current levels (response adapted to approximately $13 \%$ of initial at $574 \mu \mathrm{A}$ vs $33 \%$ of initial at $1291 \mu \mathrm{A}$ for ISI $=500 \mathrm{~ms}$ ). At interstimulus intervals of $2 \mathrm{~s}$ or more there was very little adaptation to subsequent stimulus repetitions. Based on the temporal properties related to integration and adaptation, and the assumption that independent responses are desirable, the standard stimulation protocol (ISI $=4-5 \mathrm{~s}$ ) appeared adequate for characterizing the ESR in the guinea pig.

\section{DISCUSSION AND CONCLUSION}

This study demonstrated the feasibility of intraoperative EMG recordings from the ipsilateral stapedius muscle for detecting and characterizing the stapedial reflex that is elicited via cochlear implant. In anesthetized guinea pig preparations, bipolar microwire electrodes recorded easily detectable single- and multiple-motor-unit activity with variable spike amplitudes up to $300 \mu \mathrm{V}$, with typical signal-to-noise ratios of 10-25 dB. After removing stimulus artifacts, the preferred method of EMG analysis was a relatively simple threshold-crossing algorithm because it was more robust to EMG amplitude variations and momentary segments of decreased signalto-noise ratio, as compared to a rms-energy algorithm. 
Both analysis methods resulted in similar response growth functions $(r>0.97)$, although in several animals, the rms growth function did not saturate at high stimulus levels while the threshold-crossing growth function did saturate. This discrepancy is likely due to stimulation pulse artifacts, which at high levels of stimulation sometimes persisted beyond the zeroing windows that were intended to remove them offline. This also caused the EMG signal to ride on a decaying base line between each stimulus pulse. The rms-energy measure increased as a function of these artifactual effects. The thresholdcrossing algorithm was less susceptible to the stimulation artifact because threshold crossings were not registered until after the signal decayed below threshold after a stimulus pulse. It was also not affected by small baseline shifts because it was concerned only with the spikes that crossed threshold, not the underlying base line. The threshold-crossing technique also provided sensitive measures of response latency and the temporal characteristics of muscle contraction. The high threshold levels that were used in the algorithm to identify EMG spikes (3 SD) limited the false positive detection rate, and thus provided a conservative estimate of EMG activity. The threshold-crossing algorithm is also conducive for realtime signal processing because of its computational efficiency.

The growth response functions were monotonic over a $700 \mu \mathrm{A}$ range (approximately $8 \mathrm{~dB}$ ) for the stimulation parameters used in the study $(250 / \mathrm{s}$ pulse rate, $100 \mu \mathrm{s}$ pulse widths, $500 \mathrm{~ms}$ duration, presented every 4-5 s). The visually detected contraction threshold was usually slightly higher than the threshold of the growth response function, reinforcing the observation that the stapedial EMG is a sensitive estimator of the electrical stapedial response. The normalized growth response functions were similar across animals. While the general shape of the growth functions is comparable to that of the response curves for acoustic impedance measurements in humans ${ }^{3}$ the high degree of inter-animal similarity is at odds with some of the results that show variability among cochlear implant patients. ${ }^{3,17,30}$ This discrepancy cannot be resolved at this time, but it may be explained by the same factors that influence cochlear implant performance, such as variations in the surviving spiral ganglion cells and different insertion depths due to size and ossification of the cochlear. ${ }^{11,13,20}$

Near threshold stimulus levels, latencies of the EMG response were 100-400 ms, suggesting the importance of temporal summation of the stimulus pulses at low current levels to produce detectable EMG signals. At these low stimulus levels, there was also interanimal variability and large individual standard deviations near threshold that reflect the stochastic nature of the EMG spikes. The response latencies decrease with higher stimulus levels over a range comparable to the dynamic range of the growth functions. At the highest stimulus levels the latencies decreased to $15-20 \mathrm{~ms}$, which is consistent to minimum EMG latencies of $18-20 \mathrm{~ms}$ reported for the acoustically elicited stapedial EMG in guinea pig. ${ }^{2}$ A similar sharp decrease in latency in acoustic impedance changes with increasing stimulus level has been reported for a cochlear implant user. ${ }^{16}$ With the stimulus duration of $500 \mathrm{~ms}$, temporal integration caused the EMG to reach maximum levels that varied with stimulus level. The latency to reach maximum response decreased with increasing stimulation: approximately 200 $\mathrm{ms}$ at threshold levels, but less than $50 \mathrm{~ms}$ at the highest levels. Avan et al. reported similar sharp onsets for acoustically stimulated guinea pigs. ${ }^{2}$

With long stimulus pulse trains, the EMG began to adapt after $1 \mathrm{~s}$, which is similar to the acoustic-elicited stapedial EMG adapting 1.5-2 s after stimulus onset as reported by Avan et al. ${ }^{2}$ In the present study, the adaptation to individual long duration (3s) stimuli becomes more pronounced after the first several stimulus presentations. Even for shorter-duration stimuli, interstimulus intervals affected successive responses. An interstimulus interval of $2 \mathrm{~s}$ (for $500 \mathrm{~ms}$ stimulus) removed most interactions between consecutive stimuli at low current levels, while higher current levels might require at least 4 s. Stimulation longer than this may require longer interstimulus intervals. The responses suggested adaptation over multiple time scales. The adaptation to repetitive stimuli occurs over a long time scale of 5-10 s. Adaptation to individual long-duration stimuli occurs over a shorter time scale of 1-3 s.

The causes of these characteristics are probably mainly due to adaptation in the auditory brainstem circuits that give rise to the reflex, as the stapedius muscle in many species is fatigue resistant. ${ }^{8,19,34}$ This conclusion is complicated, however, by the fact that the muscle fibers of the guinea pig stapedius are characteristic of developing fibers even in the adult. ${ }^{7}$ In any case, in order to achieve stationary EMG responses in the guinea pig, these results suggest the use of 200-500 ms stimulus pulse trains with interstimulus intervals of at least $4 \mathrm{~s}$. While the details of adaptation might be different in humans, it should be characterized and considered when constructing protocols for assessing the reflex.

The EMG recordings appear to be relatively localized to the electrode because they contained discrete motorunit firings that were often similar in shape. ${ }^{18}$ This may also be helped by the extremely low innervation ratios of the motor units in the stapedius (less than 9 in adults, ${ }^{4}$ around 16-21 in neonates, ${ }^{5}$ and $14-19$ in guinea pigs ${ }^{31}$ ). There might be concern that these localized recordings would not represent the overall activity of the muscle. Initially, we attempted to record a more global EMG signal with a monopolar electrode configuration. However, the stimulus artifact in this configuration prevented 
the successful detection of motor-unit firing between the pulses due to amplifier saturation. While there may be approaches that would circumvent the trouble of artifact in the monopolar recordings, this may be unnecessary since the bipolar recordings appeared to be proper indicators of ESR activation levels. This is aided by the fact that the stapedius muscle is quite uniform in functional composition, as innervation of the stapedius does not appear to be highly localized. ${ }^{35}$ Therefore, a relatively small sample of motor units may effectively represent the overall level of muscle activity.

Almqvist et al. ${ }^{1}$ developed a technique for detecting the ESR based on averaging the stapedial EMG (AESR) in response to brief stimulus bursts (1-4 total pulses; $<10$ ms duration). In 7 out of 12 children, using a monopolar needle electrode placed into the stapedius muscle, they were able to obtain averaged wave forms with peaks time locked to the stimulus pulses ranging from 3-30 $\mu \mathrm{V}$. Since the recorded signals were not rectified, the technique required the onset EMG wave forms to have similar shape and latency. We did not find this feature consistently in the EMG records obtained in this study. One reasonable explanation is related to differences in the recording electrode configuration. Almqvist et al. used a relatively large monopolar EMG needle electrode $(360 \mu \mathrm{m}$ diameter) that was inserted directly into the muscle. Our electrode was bipolar and considerably smaller with $50-\mu$ m-diameter tips separated by approximately $200 \mu \mathrm{m}$. As stated above, the bipolar electrode most likely recorded activity from a relatively small set of motor units in the vicinity of the electrode. These brief individual potentials varied in amplitude and time of occurrence. This was best expressed in the variability of the latency of the first EMG spike, which suggests the motor-unit potentials are not always time locked to the onset of stimulation, even at very high current levels. The large monopolar configuration, however, probably recorded wave forms that represent the summed activity from many motor units, which overall appears to have features time locked to the individual stimulus pulses. In humans stimulated with short $(50 \mathrm{~ms})$ tone bursts, similar averaged stapedial EMG signals have been reported using silver ball recording electrodes placed on the tympanic membrane or the cochlear promontory. ${ }^{36}$

Radio-frequency interference from the transmitter coil of the cochlear implant system and stimulus artifact prevented Almqvist et al. from obtaining EMG recordings during the stimulation bursts as they originally intended. ${ }^{1}$ These issues were less significant in our study. The overwhelming stimulus artifacts and radio-frequency interference that were present in monopolar recordings were sufficiently reduced in amplitude when the bipolar recording electrode was used because of common mode rejection. While stimulus artifact was still present in the recordings it did not cause amplifier recovery to extend much beyond $1 \mathrm{~ms}$ after the onset of each stimulus pulse, so that EMG spikes could be detected between them. The fact that we obtained less radio-frequency interference than Almqvist et al. may have been also due to the fact that the transmitter coil was not directly placed over the head as in the cochlear implant patients. ${ }^{1}$ The techniques we have presented have the advantage of providing a sensitive estimation of threshold while preserving the temporal properties of the response during stimuli of comparable duration to those used for fitting the device.

The bipolar microwire EMG electrode design presented here would probably need to be modified in order to apply this technique to humans. The stapedius muscle of humans is almost completely encased in the bones of the middle ear. In order to obtain surface-type recordings similar to those obtained in this study, it would be necessary to carefully drill away a portion of the pyramidal eminence as was done in the Almqvist et al. study. ${ }^{1}$ This can be a relatively risky step since an error in drilling this structure could result in damage to the underlying stapedius muscle, or even worse to the facial nerve, resulting in partial paralysis of the facial muscles. A slight modification that we are currently pursuing with some success in the rat (stapedius also encased in bone) involves slipping an electrode into the stapedial cavity along side the tendon into the space between the bone and the stapedius. The electrode is bipolar with the exposed tips of two wires fixed together such that the contacts are offset by $200-400 \mu \mathrm{m}$. Relatively strong EMG signals have been obtained with this type of electrode in acute experiments. It is yet unclear how well this configuration will work, but chronic studies will reveal new information regarding the effect a long-term implanted electrode might have on the stapedius muscle. Placing an electrode on the tendon or the bone near the tendon exit may be another possibility. However, significant signal degradation would be expected due to the distance from the signal sources. Recording such small signals might not be possible with the current technology of onboard amplifier and telemetry systems available in cochlear implants today.

It is important to note that the stapedius reflex is known to be significantly affected by the level and type of anesthetic. ${ }^{12,33}$ In general, anesthesia tends to shift stapedial reflex threshold upward, ${ }^{12}$ but it is extremely difficult to predict postoperative responses from intraoperative results. In addition, spontaneous activity may be expected in unanesthetized animals ${ }^{9}$ and humans, ${ }^{10}$ which might make the problem of threshold detection more challenging, but not intractable with signal processing approaches similar to those described. Caution must be taken in generalizing the results presented here to different species and/or anesthetic regimens. Future stud- 
ies will attempt to record the EMG response from awake animals to eliminate anesthesia effects.

The feasibility of recording real-time stapedial EMG under ipsilateral electrical simulation was demonstrated in the guinea pig model. Relatively simple processing strategies were employed to sufficiently characterize the ESR. With further development aimed at obtaining chronic recordings in animal models, these techniques may eventually be applied to human cochlear implant recipients as a guide in setting comfort levels.

\section{ACKNOWLEDGMENTS}

This project was supported by the National Science Foundation (BES-9624636), and by a graduate fellowship from the Whitaker Foundation. The authors acknowledge Jon K. Shallop (Mayo Clinic, Rochester, $\mathrm{MN}$ ) for insightful comments and for guidance in preliminary experiments. The authors also extend special thanks to Gabriel Brat, Dave Brown, and Dianne LoBaido for providing valuable technical assistance.

\section{REFERENCES}

${ }^{1}$ Almqvist, B., S. Harris, and J. K. Shallop. Objective intraoperative method to record averaged electromyographic stapedius muscle reflexes in cochlear implant patients. Audiology 39:146-152, 2000.

${ }^{2}$ Avan, P., D. Loth, C. Menguy, and M. Teyssou. Hypothetical roles of middle ear muscles in the guinea pig. Hear. Res. 59:59-69, 1992.

${ }^{3}$ Battmer, R. D., R. Laszig, and E. Lehnhardt. Electrically elicited stapedius reflex in cochlear implant patients. Ear Hear. 11:370-374, 1990.

${ }^{4}$ Blevins, C. E. Innervation patterns of the human stapedius muscle. Arch. Otolaryngol. 86:136-142, 1967.

${ }^{5}$ Blevins, C. E. Motor units in the stapedius muscle. Arch. Otolaryngol. 87:249-254, 1968.

${ }^{6}$ Borg, E., S. A. Counter, and G. Rosler. Theories of middleear muscle function. In: The Acoustic Reflex: Basic Principles and Clinical Application, edited by S. Silman. Orlando, FL: Academic, 1984, pp. 63-99.

${ }^{7}$ Burgener, J., and R. Mayr. Guinea pig stapedius muscle. A histochemical, light, and electron microscopic study. Anat. Embryol. 161:65-81, 1980.

${ }^{8}$ Dammeijer, P. F., H. van Mameren, P. van Dijk, A. F. Moorman, P. Habets, J. J. Manni, and J. Drukker. Stapedius muscle fiber composition in the rat. Hear. Res. 141:169-179, 2000.

${ }^{9}$ Eliasson, A., and L. Gisselsson. Electromyographic studies of the middle ear muscles of the cat. Electroencephalogr. Clin. Neurophysiol. 195:399-406, 1955.

${ }^{10}$ Fisch, U., and G. V. Schulthess. Electromyographic studies on the human stapedial muscle. Arch. Otolaryngol. 56:287297, 1963.

${ }^{11}$ Geier, L., J. Gilden, C. M. Luetje, and H. E. Maddox III. Delayed perception of cochlear implant stimulation in children with postmeningitic ossified cochleae. Am. J. Otolaryngol. 14:556-561, 1993.

${ }^{12}$ Gnadeberg, D., R. D. Battmer, E. Lullwitz, R. Laszig, U. Dybus, and T. Lenarz. Effect of anesthesia on the intraopera- tive elicited stapedius reflex. Laryngorhinotologie 73:132135, 1994.

${ }^{13}$ Hartrampf, R., M. C. Dahm, R. D. Battmer, D. Gnandeberg, A. Strauss-Schier, U. Rost, and T. Lenarz. Insertion depth of the Nucleus electrode array and relative performance. Ann. Otol. Rhinol. Laryngol. 166:277-280, 1995.

${ }^{14}$ Hodges, A. V., T. J. Balkany, R. A. Ruth, P. R. Lambert, S. Dolan-Ash, and J. J. Schloffman. Electrical middle ear muscle reflex: Use in cochlear implant programing. Otolaryngol.-Head Neck Surg. 117:255-261, 1997.

${ }^{15}$ Hodges, A. V., S. Butts, S. Dolan-Ash, and T. J. Balkany. Using electrically evoked auditory reflex thresholds to fit the CLARION cochlear implant. Ann. Otol. Rhinol. Laryngol. 177:64-68, 1999.

${ }^{16}$ Jerger, J., H. Jenkins, R. Fifer, and D. Mecklenburg. Stapedius reflex to electrical stimulation in a patient with a cochlear implant. Ann. Otol. Rhinol. Laryngol. 95:151-157, 1986.

${ }^{17}$ Jerger, J., T. A. Oliver, and R. A. Chmiel. Prediction of dynamic range from stapedius reflex in cochlear implant patients. Ear Hear. 9:4-8, 1988.

${ }^{18}$ Loeb, G. E., and C. Gans. Electromyography for Experimentalists. Chicago: University of Chicago Press, 1986.

${ }^{19}$ Lyon, M. J., and L. T. Malmgren. A histochemical characterization of muscle fiber types in the middle ear muscles of the cat. 1. The stapedius muscle. Arch. Otolaryngol. 94:99-109, 1982.

${ }^{20}$ Otte, J., H. F. Schunknecht, and A. G. Kerr. Ganglion cell populations in normal and pathological human cochleae. Implications for cochlear implantation. Laryngoscope 88:12311246, 1978.

${ }^{21}$ Pilz, P. K., J. Ostwald, A. Kreiter, and H. U. Schnitzler. Effect of the middle ear reflex on sound transmission to the inner ear of rat. Hear. Res. 105:171-182, 1997.

${ }^{22}$ Roberts, S. Speech-processor fitting for cochlear implants. In: Cochlear Implants: A Practical Guide, edited by H. R. Cooper. London, U.K.: Whurr, 1991, pp. 201-218.

${ }^{23}$ Shallop, J. K. Objective electrophysiological measures from cochlear implant patients. Ear Hear. 14:58-63, 1993.

${ }^{24}$ Shallop, J. K. Objective measurements and the audiological management of cochlear implant patients. Adv. Oto-RhinoLaryngol. 53:85-111, 1997.

${ }^{25}$ Shallop, J. K., and K. R. Ash. Relationships among comfort levels determined by cochlear implant patient's selfprograming, audiologist's programing, and electrical stapedius reflex thresholds. Ann. Otol. Rhinol. Laryngol. 166:175176, 1995.

${ }^{26}$ Sheppard, S. Fitting and programming the external system. In: Cochlear Implants for Young Children: The Nottingham Approach to Assessment and Rehabilitation, edited by B. McCormick, S. Archbold, and S. Sheppard. London, U.K.: Whurr, 1994, pp. 140-165.

${ }^{27}$ Spivak, L. G., and P. M. Chute. The relationship between electrical acoustic reflex thresholds and behavioral comfort levels in children and adult cochlear implant patients. Ear Hear. 15:184-192, 1994.

${ }^{28}$ Stephan, K., and K. Welzl-Muller. Postoperative stapedius reflex tests with simultaneous loudness scaling in patients supplied with cochlear implants. Audiology 39:13-18, 2000.

${ }^{29}$ Stephan, K., K. Welzl-Muller, and H. Stiglbrunner. Stapedius reflex threshold in cochlear implant patients. Audiology 27:227-233, 1988.

${ }^{30}$ Stephan, K., K. Welzl-Muller, and H. Stiglbrunner. Stapedius reflex growth function in cochlear implant patients. Audiology 29:46-54, 1990.

${ }^{31}$ Strutz, J., G. Munker, and C. Zollner. The motor innervation 
of the tympanic muscles in the guinea pig. Oto-RhinoLaryngol. 254:108-111, 1988.

${ }^{32}$ Svirsky, M. A. The cochlear implant field is slowly approach the time when electrophysiologic measures will be widely used in cochlear implant fitting. Ear Hear. 21:84, 2000.

${ }^{33}$ van den Borne, S., A. F. Snik, L. H. Mens, J. P. Brokx, and P. van den Broek. Stapedius reflex measurements during surgery for cochlear implantation in children. Am. J. Otolaryngol. 17:554-558, 1996.

${ }^{34}$ Veggetti, A., F. Mascarello, and E. Carpene. A comparative histochemical study of fiber types in middle ear muscles. $J$.
Anat. 135:333-352, 1982.

${ }^{35}$ Wiener-Vacher, S. R., J. J. Guinan, Jr., J. B. Kobler, and B. E. Norris. Motoneuron axon distribution in the cat stapedius muscle. Hear. Res. 133:139-148, 1999.

${ }^{36}$ Yagi, N., and H. Nakatani. Stapedial electromyograms recorded by electrocochleography. Ann. Otol. Rhinol. Laryngol. 97:87-91, 1988.

${ }^{37}$ Zakrisson, J. E., E. Borg, and S. Blom. The acoustic impedance change as a measure of stapedius muscle activity in man. A methodological study with electromyography. Acta Otolaryngol. 78:357-364, 1974. 